\title{
Algorithm and Hardware Implementation of Image Haze Removal Combining with Sky Recognition
}

\author{
Xiaobo ZHOU, Xiaodong FAN* \\ School of Electric and Information Engineering \\ Beijing Jiaotong University \\ Beijing, China \\ *E-mail: fxd8081@163.com \\ +* Corresponding author
}

\begin{abstract}
When a picture is taken in the haze, the picture is abstract and much information is hidden by the haze. In this paper, on the basis of the dark channel prior algorithm, dehazing of hazy images is carried out. At the same time, given defects of the dark channel prior algorithm, namely halo, color lumps and color cast etc. on the sky area after dehazing, sky recognition algorithm and guided filter algorithm are integrated together to dehaze. The sky recognition algorithm can distinguish the sky region effectively. And high-precision transmissivity parameter can be obtained using guided filter algorithm. The whole algorithm has been verified in both the $C$ language simulation environment and the Matlab simulation environment. The hardware implementation is completed on the self-designed FPGA hardware platform. Running results show that the operation of the algorithm proposed in this paper is stable and it can carry out image dehazing effectively.
\end{abstract}

Keywords-dark channel prior; guided image filter; sky recognition; fpga

\section{INTRODUCTION}

In the recent decade, haze pollution has become one of the most important problems of environmental pollution in many cities. Haze can cause quality reduction of images [1], low definition of security monitoring systems and precision decline of remote sensing monitoring systems etc. In order to obtain more information from hazy images, researchers need to carry out dehazing processing of hazy images first.

Now there are two main classes of the image dehazing technology, namely image enhancement and image restoration. Image enhancement enlarges the whole contrast of an image [2,3] without any differentiation. Though it shows certain dehazing effects, it also increases the noise of the image. Based on physical models proposed by McCartney [4], image restoration gets dehazing formulas by reverse calculation in line with the principle and then calculates parameters of physical models to restore into fogless images. The dark channel prior algorithm put forward by Kaiming $\mathrm{He}$ (2011) [5] is one of the most effective image restoration technologies. In this paper, on the basis of the dark channel prior algorithm and in combination with the sky recognition algorithm, dehazing is conducted. The algorithm studied in this paper is realized on the self-designed FPGA hardware platform to complete dehazing processing of hazy images, obtaining good dehazing effects.

\section{THEORY RESEARCH}

\section{A. Dark Channel Prior Algorithm}

In computer vision and computer graphics fields, a physical model shown in formula (1) is usually used to describe influences of haze on images [5]:

$$
\mathbf{I}(\mathbf{x})=\mathbf{J}(\mathbf{x}) \cdot t(\mathbf{x})+\mathbf{A} \cdot(1-t(\mathbf{x}))
$$

Where $\mathrm{I}(\mathrm{x})$ is a hazy image, $\mathrm{J}(\mathrm{x})$ is an outputting fogless image, A expresses the overall atmospheric light and $t(x)$ is the medium transmissivity. With the increase of $\mathrm{t}(\mathrm{x})$, more raw physical information is received by photography instrument and naked eyes with less haze. Now the only solving condition of formula (1) is $\mathrm{I}(\mathrm{x})$, so the equation has many solutions. The variant of formula (1) is shown in formula (2) where c refers to anyone of the three channels of RGB [5].

$$
\frac{I^{c}(\mathbf{x})}{A^{c}}=t(\mathbf{x}) \frac{J^{c}(\mathbf{x})}{A^{c}}+1-t(\mathbf{x})
$$

In dark channel of an image, the minimum value of RGB channels of neighboring pixels is taken as the value of the dark channel of this pixel [5], as shown in formula (3).

$$
J_{d a r k}(\mathbf{x})=\min _{y \in \Omega(\mathrm{x})}\left(\min _{c \in\{r, g, b\}} J^{c}(\mathbf{y})\right)
$$

Where $\Omega(\mathrm{x})$ is a neighborhood centering on pixel $\mathrm{x}$. As is shown in formula (4), dark channel prior means that there always exists some pixels whose value approaching to zero $[5,6]$.

$$
J_{\text {dark }} \rightarrow 0
$$

After formula (4) is substituted into formula (2), formula (5) is obtained by simplification [5]. 


$$
t(\mathbf{x})=1-\min _{y \in \Omega(\mathrm{x})}\left(\min _{c} \frac{I^{c}(\mathbf{y})}{A^{c}}\right)
$$

As a result, $\mathrm{t}(\mathrm{x})$ of each pixel can be obtained. Under normal circumstances, when people see objects at a distance, slight fog can express different depths of field. Therefore, in formula (6), t 0 , the minimum value of $t$, is utilized to simulate effects observed by naked eyes [5].

$$
\mathbf{J}(\mathbf{x})=\frac{\mathbf{I}(\mathbf{x})-\mathbf{A}}{\max \left(t(\mathbf{x}), t_{0}\right)}+\mathbf{A}
$$

However, the transmissivity parameter $\mathrm{t}$ with low precision obtained above of several adjacent pixels are same. In areas where depth differences of photographic fields are large, problems of image blurring and color cast can be caused easily after processing. Hence, some scholars utilized the method of soft matting before [7]. Thought it had good effects, its calculation is huge, causing a slow processing speed. In view of the low precision of transmissivity parameter $\mathrm{t}$ mentioned above, Kaiming He proposed a new algorithm called guided image filtering [8] to recalculate parameter $t$. The main parameters of guided filter are image $\mathrm{p}$ of filter input, guided image $\mathrm{I}$ and outputting image $\mathrm{q}$. As for a random pixel i, the filtering result is shown in formula (7) [8]:

$$
q_{i}=\sum_{j} W_{i j}(I) \cdot p_{j}
$$
[8].

And then formula (7) can be simplified to formula (8)

$$
q_{i}=a_{k} I_{i}+b_{k}, \forall i \in \omega_{k}
$$

In order to maintain the linear relationship between $p$ and $\mathrm{q}$, minimization of the loss function within filter window $\omega \mathrm{k}$ is needed. Formula (9) is a linear ridge regression model [8], $\varepsilon$ is a regular parameter used for weakening adverse effects when ak is relatively big.

$$
E\left(a_{k}, b_{k}\right)=\sum_{i \in \omega_{k}}\left(\left(a_{k} I_{i}+b_{k}-p_{i}\right)^{2}+\varepsilon \cdot a_{k}^{2}\right) .
$$

By finding the optimal solution of formula (9), solutions of ak and bk can be obtained [8] and are shown in formula (10) and formula (11).

$$
\begin{gathered}
a_{k}=\frac{\frac{1}{|\omega|} \sum_{i \in \omega_{k}} I_{i} p_{i}-\mu_{k} \bar{p}_{k}}{\sigma_{k}^{2}+\epsilon} \\
b_{k}=\bar{p}_{k}-a_{k} \mu_{k}
\end{gathered}
$$

Parameter ak and bk are substituted into formula (8) to obtain the outputting image. However, when it refers to all $\omega \mathrm{k}$ covering pixel $\mathrm{i}$, different $\omega \mathrm{k}$ can get different qi. A simple processing method is to get the mean of all qi [8], as shown in formula (12).

$$
q_{i}=\frac{1}{|\omega|} \sum_{k \mid i \in \omega_{k}}\left(a_{k} I_{i}+b_{k}\right) .
$$

Image I expresses a gray-scale image and image $\mathrm{p}$ expresses a low-precision transmissivity image. By guided image filtering processing of image $\mathrm{p}$, a high-precision transmissivity image can be obtained. The average of pixels with top $0.1 \%$ of brightness in Jdark are counted as value A. By substituting $\mathrm{A}$ and that has been obtained into formula (6), $\mathrm{J}(\mathrm{x})$, namely the fogless image, is obtained after simplification.

\section{B. Sky Recognition Algorithm}

The dark channel prior algorithm works well when it deals with hazy images without the sky region. However, if it is used to process an image containing the sky, large areas of halo, color blocks and color cast etc. will appear, leading to a poor dehazing effect.

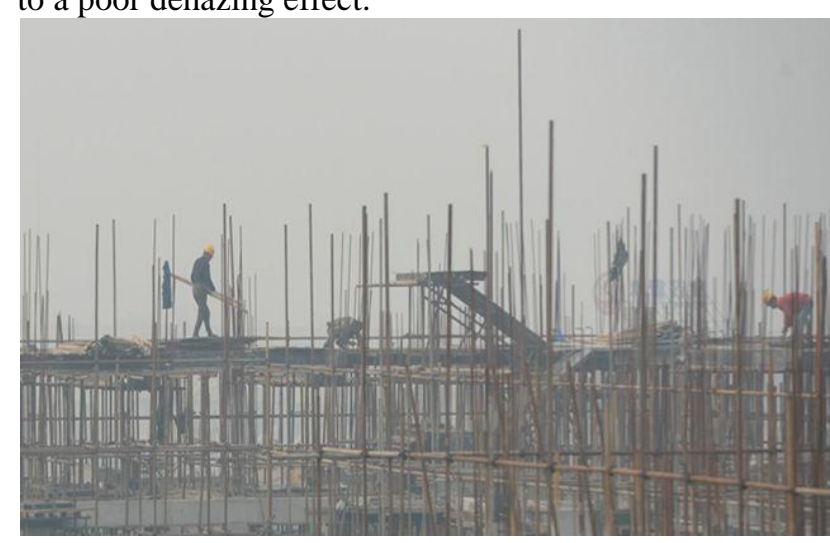

Figure 1. Original hazy image

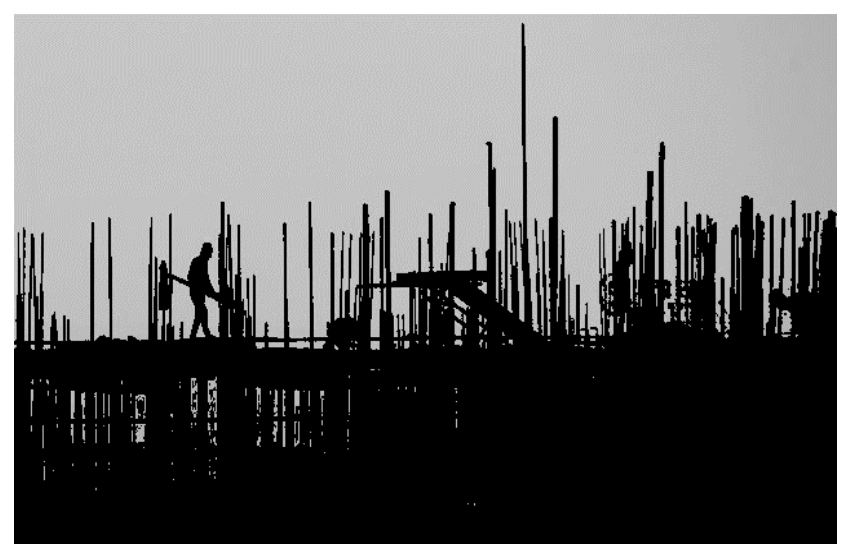

Figure 2. Binarization of haze image

Figure 2 is a result of binarization processing of Figure 1. It can be seen that the binarization processing can effectively distinguish the bright parts of an image. Therefore, in this paper, via sky recognition algorithm, pixels of the sky region of an image are recognized first and dehazing processing of the sky part and the non-sky part are 
carried out respectively. In most images, the sky region is characterized by close values of RGB channels among adjacent pixels. The recognition threshold is defined as A0 [9]. In dark channel image Idark, if a pixel's value is greater than $\mathrm{A} 0$, its corresponding position in the binary image is marked as 1 . If it is not, that is marked as 0 .

C. Flowchart of the Dehazing Algorithm

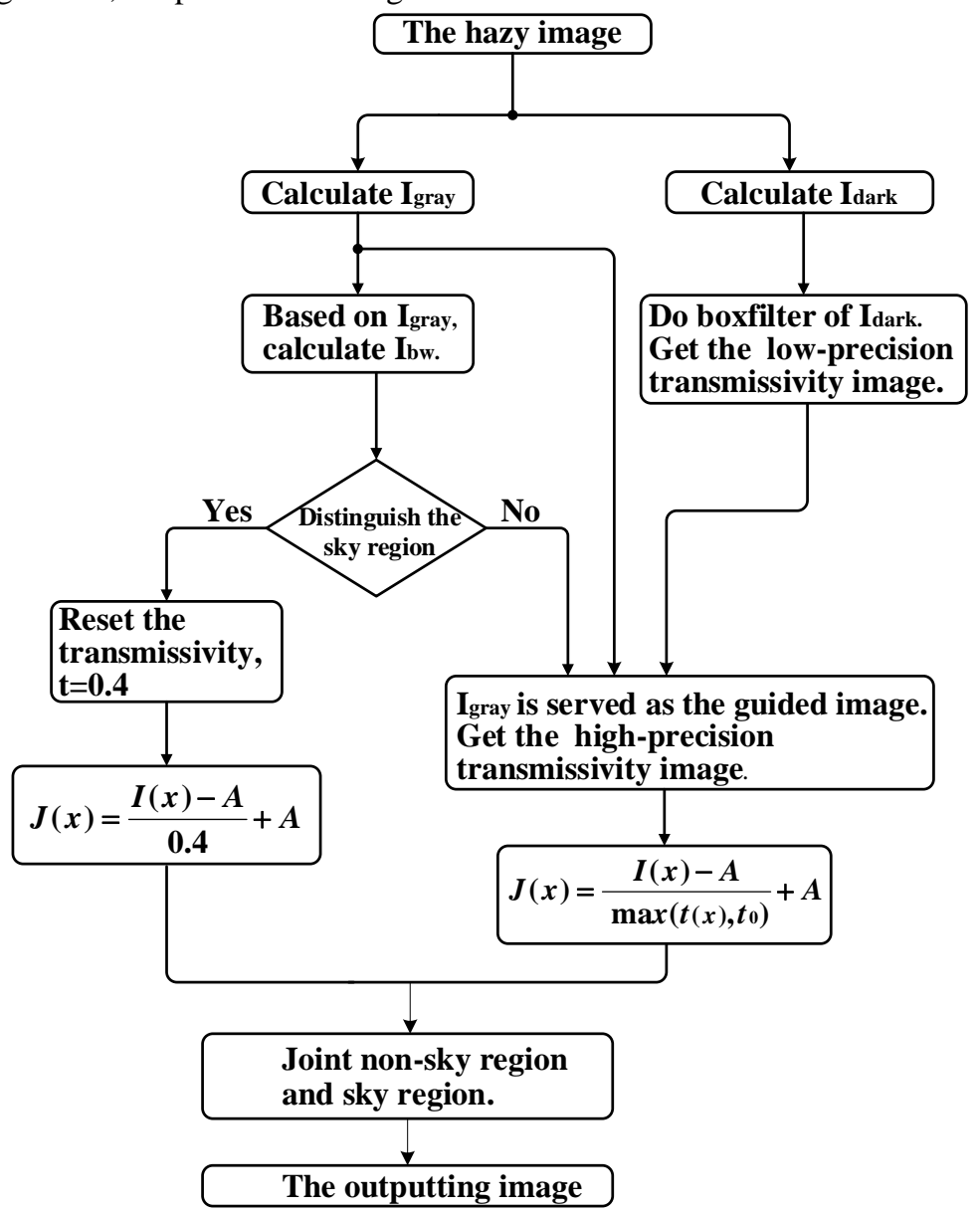

Figure 3. Overall flow chart of the dehazing algorithm

The overall process of the dehazing algorithm in this paper is shown in Figure 3. For an obtained hazy image, the first step is to calculate its Idark and low-precision transmissivity image. At the same time, the input image is transformed into a gray-scale image. The solution of $\mathrm{A}$ is simplified into calculating the maximum value of pixels of Idark. The second step is to compare the value of each pixel of Idark with A0 and obtain binary image Ibw. The third step is to process the low-precision transmissivity image obtained in step one with guided filtering processing and obtain a high-precision transmissivity image.
In the fourth step, Ibw is mapped to the original image to identify the sky area. As the transmissivity of the entire sky area is close, transmissivity parameter $t$ of the sky area is a fixed value of 0.4 during calculation. By substituting it into the next equation, a dehazing image of the sky part can be obtained. For the non-sky area, in the fifth step, value A and the high-precision precision transmissivity parameter $\mathrm{t}$ are substituted into formula (6) to obtain a dehazing image. Finally, by jointing images got during steps four and five, a complete dehazing image is obtained. 
D. Overall Design of the Hardware Platform

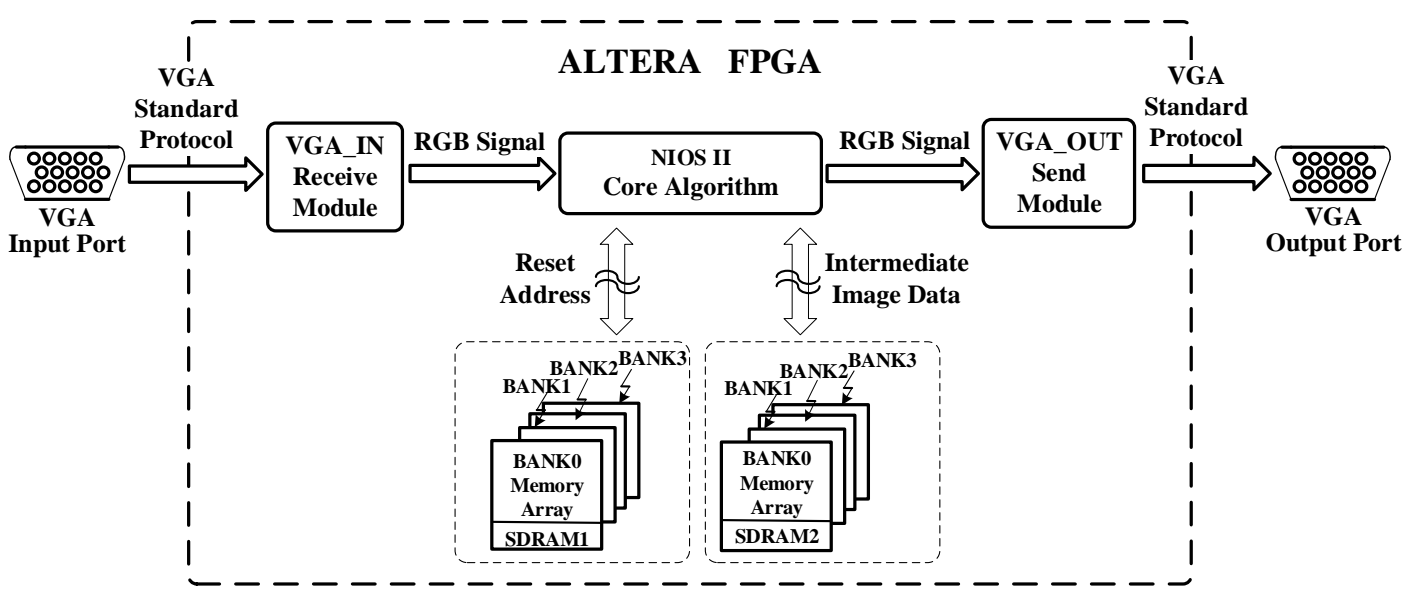

Figure 4. The structure of hardware design

In this paper, the dehazing algorithm is implemented on the self-designed FPGA video enhancement platform. This platform mainly includes the core chip, ALTERA FPGA Cyclone IV EP4CE40F23C7, its peripheral circuits, AD chip AD9984, which is responsible for video input conversion, DA chip ADV7123, which is responsible for video output conversion, VGA graphics interface which takes charge of video input port and output port and two groups of SDRAM of the Micron MT48LC16M16A2P-6A series.

The overall hardware structure is shown in Figure 4. SDRAM1 supplies the resetting address space of NIOS II and cache images delivered from VGA_IN module. The main function of SDRAM2 is loading core algorithm modules of NIOS II and serving as a cache area of intermediate results of the algorithm. The receiving module named VGA_IN firstly peel off data from the standard VGA protocol to obtain data of RGB channels. RGB data output by VGA_IN receive module is saved on SDRAM1 for caching and be read by NIOS II in the next step. After reading the original image from SDRAM1, algorithms of guided filter and sky recognition are implemented. And then all intermediate results are stored in SDRAM2 temporally. Intermediate results include gray-scale images, low-precision transmissivity images, high-precision transmissivity images, parameters of guided filter, dehazing images of sky areas, dehazing images of non-sky areas and jointed images. The outputting image of dehazing processing in NIOS II, what is only RGB data without any video protocol. Dehazing images are output to VGA_OUT send module. And then image data and the VGA standard protocol are coupled and output to VGA hardware port.

\section{ANALYSIS OF DEHAZING RESULTS}

The processing results of every algorithm step are shown in Figure 5.

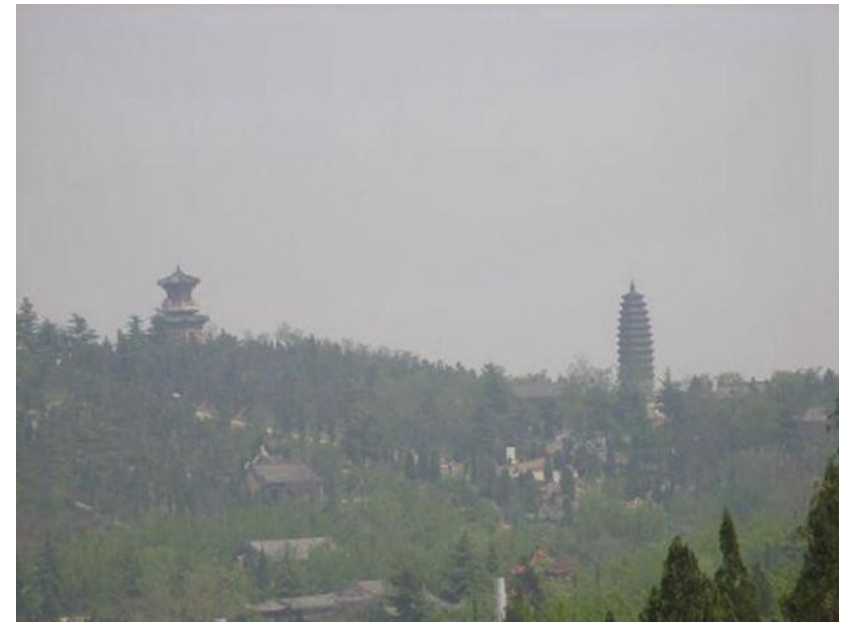

(a) Inputting haze image

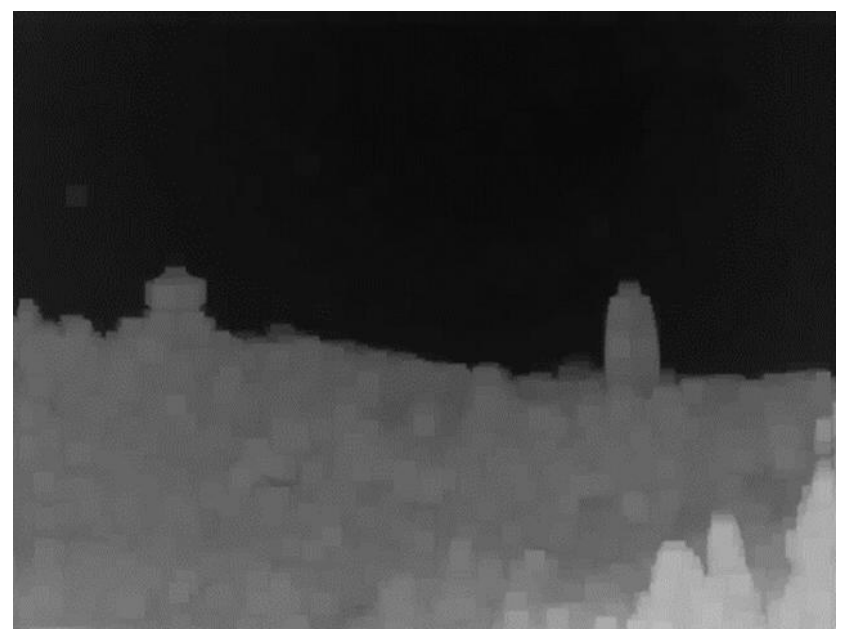

(b) $t(x)$ with low-precision 


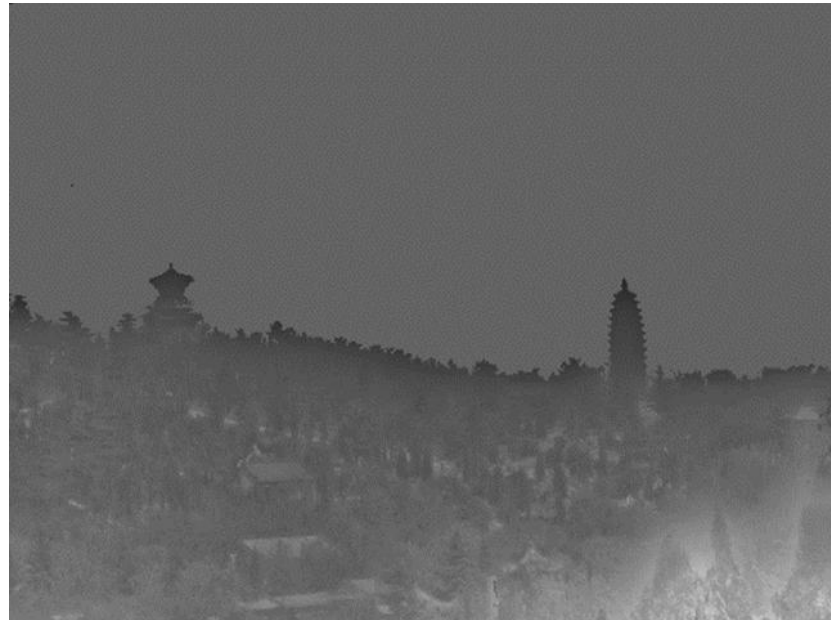

(c) $t(x)$ with high-precision

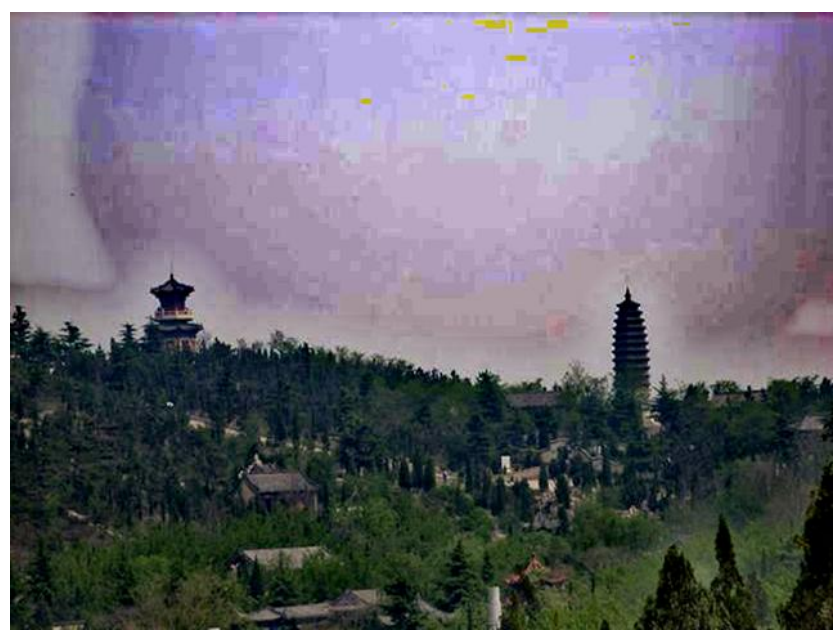

(d) Without sky recognition

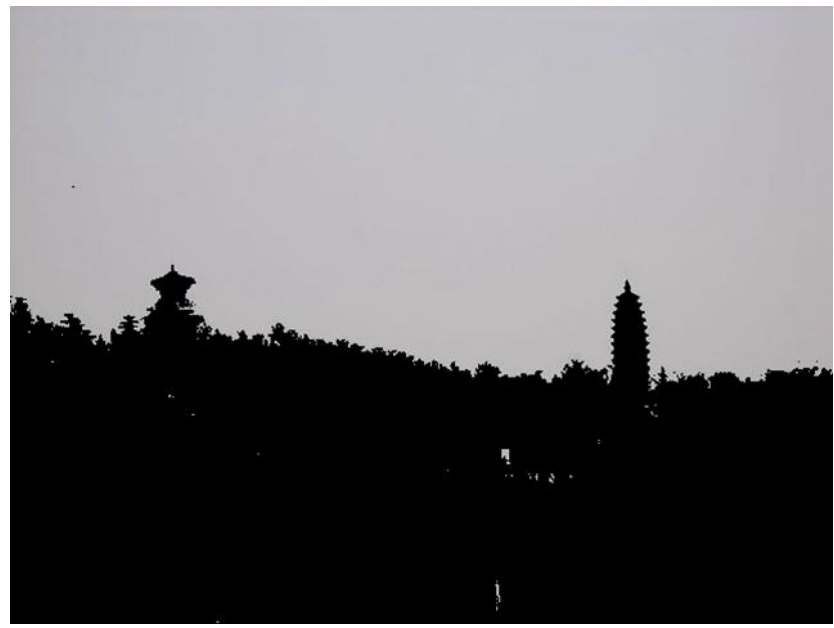

(e) Sky recognition

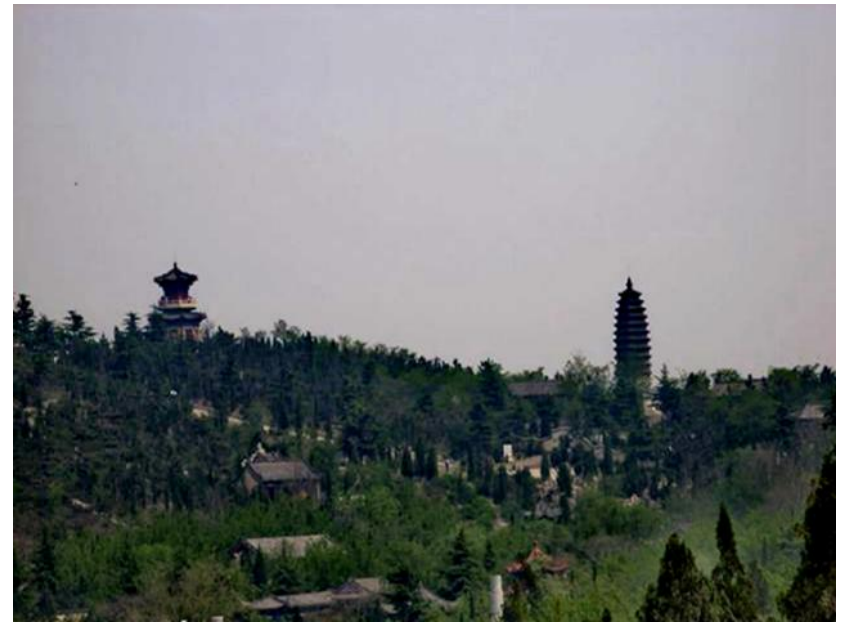

(f) Final outputting image

Figure 5. Results of every processing step

Figure 5 (a) is an input hazy image. It can be seen that the haze obviously impacts the image quality. Figure 5(b) is a low-precision transmissivity image and Figure 5(c) is a high-precision transmissivity image calculated by guided filtering. It is obvious that the accuracy is relatively enhanced. Figure 5(d) shows a dehazing effect of guided filter on the basis of Figure 5(c). In Figure 5(d), more color and contrast information have been restored, but the sky area shows halo, color lumps and color cast.

Figure 5(e) is a binary image of the sky area after the sky recognition algorithm processing. It can be seen that it recognizes the sky area effectively with high precision. Figure 5(f) is the final dehazing image by jointing dehazing images of sky and non-sky areas. Compared with Figure 5(d), Figure 5(f) has a better dehazing effect. The sky area is without halo, color blocks or color cast. On the whole, the dehazing algorithm showed a good effect and its practical results running on hardware platform are consistent with software simulation results.

\section{CONCLUSIONS}

On the basis of the dark channel prior algorithm, this paper analyzed its advantages and implementation methods. At the same time, given defects of guided filter, including halo, color blocks and color cast on the sky area, an idea of dehazing in combination with sky recognition was put forward. And the sky recognition algorithm can recognize the sky region effectively with good recognition effects and reach high recognition precision on the boundary between sky and non-sky areas. The entire dehazing algorithm is designed completely and then verified through the $\mathrm{C}$ language simulation environment and the Matlab simulation environment. In addition, after actualizing the algorithm in this paper on the self-designed FPGA hardware development platform, it works effectively and stably.

However, when running on the FPGA hardware platform, it is difficult to reach the speed level of millisecond running on NIOS II. Therefore, a hardware accelerator is needed for the core algorithm to further enhance the running speed of the hardware platform. 


\section{REFERENCE}

[1] Tan R T. Visibility in bad weather from a single image[C]// Computer Vision and Pattern Recognition, 2008. CVPR 2008. IEEE Conference on. IEEE, 2008:1-8.

[2] Kim J H, Sim J Y, Kim C S. Single image dehazing based on contrast enhancement[C]// IEEE International Conference on Acoustics, Speech and Signal Processing. IEEE, 2011:1273-1276.

[3] Narasimhan S G, Nayar S K. Contrast restoration of weather degraded images [J]. IEEE Transactions on Pattern Analysis \& Machine Intelligence, 2003, 25(6):713-724.

[4] Mccartney E J, Jr F F H. Optics of the Atmosphere: Scattering by Molecules and Particles[J]. Journal of Modern Optics, 1977, 14(7):698-699.

[5] He K, Sun J, Tang X. Single image haze removal using dark channel prior $[\mathrm{C}] / /$ Computer Vision and Pattern Recognition, 2009. CVPR 2009. IEEE Conference on. IEEE, 2011:1956-1963.

[6] [6] Sun W, Da-Jian L I, Liu H J, et al. Fast single image fog removal based on atmospheric scattering model[J]. Optics \& Precision Engineering, 2013, 21(4):1040-1046. In Chinese.

[7] Kun L I, Lan S Y, Zhang J W, et al. An Improved Algorithm of Haze Removal Based on Dark Channel Prior[J]. Computer Technology \& Development, 2015. In Chinese.

[8] He K, Sun J, Tang X. Guided Image Filtering[M]// Computer Vision - ECCV 2010. Springer Berlin Heidelberg, 2010:1397-1409.

[9] Wang G, Ren G, Jiang L, et al. Single Image Dehazing Algorithm Based on Sky Region Segmentation[J]. Information Technology Journal, 2013, 12(6):1168-1175. 